University of Texas Rio Grande Valley

ScholarWorks @ UTRGV

$1-1-2010$

\title{
EFFECTS OF $\boldsymbol{\alpha}$-ELEMENT ENHANCEMENT AND THE THERMALLY PULSING-ASYMPTOTIC GIANT BRANCH ON SURFACE BRIGHTNESS FLUCTUATION MAGNITUDES AND BROADBAND COLORS
}

Hyun Chul Lee

Guy Worthey

John P. Blakeslee

Follow this and additional works at: https://scholarworks.utrgv.edu/pa_fac

Part of the Astrophysics and Astronomy Commons

\section{Recommended Citation}

Hyun Chul Lee, et. al., (2010) Effects of Ît-Element enhancement and the thermally pulsing-asymptotic giant branch on surface brightness fluctuation magnitudes and broadband colors.Astrophysical Journal710:1421. DOI: http://doi.org/10.1088/0004-637X/710/1/421

This Article is brought to you for free and open access by the College of Sciences at ScholarWorks @ UTRGV. It has been accepted for inclusion in Physics and Astronomy Faculty Publications and Presentations by an authorized administrator of ScholarWorks @ UTRGV. For more information, please contact justin.white@utrgv.edu, william.flores01@utrgv.edu. 


\title{
EFFECTS OF $\alpha$-ELEMENT ENHANCEMENT AND THE THERMALLY PULSING-ASYMPTOTIC GIANT BRANCH ON SURFACE BRIGHTNESS FLUCTUATION MAGNITUDES AND BROADBAND COLORS
}

\author{
Hyun-Chul LeE ${ }^{1,2}$, Guy Worthey ${ }^{1}$, and John P. BlakesLeE ${ }^{3}$ \\ ${ }^{1}$ Department of Physics and Geology, University of Texas-Pan American, Edinburg, TX 78539, USA \\ ${ }^{2}$ Department of Physics and Astronomy, Washington State University, Pullman, WA 99164-2814, USA; leeh@utpa.edu \\ ${ }^{3}$ NRC Herzberg Institute of Astrophysics, Victoria, BC V9E 2E7, Canada \\ Received 2009 February 6; accepted 2009 December 15; published 2010 January 19
}

\begin{abstract}
We investigate the effects of $\alpha$-element enhancement and the thermally pulsing-asymptotic giant branch (TP-AGB) stars on the surface brightness fluctuation (SBF) magnitudes and broadband colors of simple stellar populations and compare to the empirical calibrations. We consider a broad range of ages and metallicities using the recently updated Teramo BaSTI isochrones. We find that the $\alpha$-element-enhanced $I$-band SBF magnitudes are about 0.35 mag brighter and their integrated $V-I$ colors are about 0.02 mag redder, mostly because of oxygen-enhancement effects on the upper red giant branch and AGB. We also demonstrate, using both the Teramo BaSTI and Padova isochrones, the acute sensitivity of SBF magnitudes to the presence of TP-AGB stars, particularly in the near-IR, but in the $I$ band as well. Empirical SBF trends therefore hold great promise for constraining this important but still highly uncertain stage of stellar evolution. In a similar vein, non-negligible disparities are found among several different models available in the literature due to intrinsic model uncertainties.
\end{abstract}

Key words: galaxies: stellar content - stars: abundances - stars: evolution

Online-only material: color figures

\section{INTRODUCTION}

The surface brightness fluctuation (SBF) method, which measures the intrinsic pixel-to-pixel intensity variance in a galaxy image, is widely used as one of the most powerful distance indicators as well as a useful tool for probing stellar populations in the integrated light of early-type galaxies and spiral bulges. It is now a well-known fact that SBF magnitudes vary as a function of galaxy colors (e.g., Tonry et al. 2001; Jensen et al. 2003). For instance, empirical relations show that galaxies with redder $V-I$ colors have fainter $I$-band SBF magnitudes (e.g., Tonry 1991; Tonry et al. 1997, 2001). Moreover, there have been some suggestions that bluer dwarf elliptical galaxies have a shallower slope in the $V-I$ versus $I$-band SBF magnitude diagram compared to the redder, massive galaxies (e.g., Blakeslee et al. 2001; Mei et al. 2005; Mieske et al. 2006).

From the observational side, it is relatively well established that the red massive early-type galaxies have at least some lighter elements enhanced relative to Fe-peak elements by about 0.3-0.4 dex (e.g., Worthey et al. 1992; Lee \& Worthey 2005). This abundance pattern may resemble that of halo $\alpha$-element enhancement, although this has not been thoroughly proven (Worthey 1998). There are several theoretical spectrophotometric studies that consider $\alpha$-enhancement in order to address those observations (e.g., Thomas et al. 2003; Lee \& Worthey 2005; Coelho et al. 2007; Schiavon 2007; Lee et al. 2009). However, only solar-scaled SBF model's predictions have been calculated in the past (Worthey 1993, 1994; Liu et al. 2000; Blakeslee et al. 2001; Lee 2001; Mei et al. 2001; Cantiello et al. 2003; Mouhcine et al. 2005; Raimondo et al. 2005; Marín-Franch \& Aparicio 2006).

Moreover, because of the nature of the SBF method, which is far more sensitive to the brighter stars compared to the integrated photometry, thermally pulsing-asymptotic giant branch (TPAGB) phase is predominately important (e.g., Liu et al. 2000; Mouhcine et al. 2005; Raimondo et al. 2005). The TP-AGB phase is the last stage of AGB evolution. After the high-mass main-sequence (MS) stars and supergiants fade away, it is AGB stars that dominate the integrated bolometric light until full-fledged red giant branch (RGB) stars are looming. After $t>2$ Gyr, the RGB tip becomes nearly as bright as the AGB tip but is much more numerously populated, by nearly a factor of 10 (e.g., Ferraro et al. 2004; Mucciarelli et al. 2009). The recent development of detailed studies of TP-AGBs (e.g., Maraston 2005; Maraston et al. 2006; Lee et al. 2007b; Marigo et al. 2008) can be thoroughly inspected with observations by the SBF method. In this study, we explore the question of how the $\alpha$-elements (e.g., O, Mg, Si, S, Ca, Ti) and TP-AGBs affect the galaxy colors and SBF magnitudes.

\section{MODELS}

The present stellar population synthesis models are based upon the Teramo BaSTI isochrones ${ }^{4}$ (Pietrinferni et al. 2004, 2006; Cordier et al. 2007). The models we compute are all single starbursts, characterized by a single age and metallicity. In reality, galaxies are generally composed of mixtures of stellar populations with a range of ages and metallicities.

We have, however, deliberately employed a simplified (i.e., single burst) star formation prescription in this study so that we can explore the importance of varying one parameter, namely the $\alpha$-element enhancement. In this regard, our single-burst models approximately represent the luminosity weighted mean age and metallicity for the stellar systems in question. Following our previous models (Lee et al. 2007b, 2009), we employ the standard Salpeter (1955) initial mass function (IMF). The lowmass cutoff is $0.5 M_{\odot}$ as given in the Teramo BaSTI isochrones. We do not consider the stochastic nature of the TP-AGB in this study. We merely calculate the SBF magnitudes and the broadband colors using the isochrones as they are available

\footnotetext{
4 http://193.204.1.62/index.html
} 

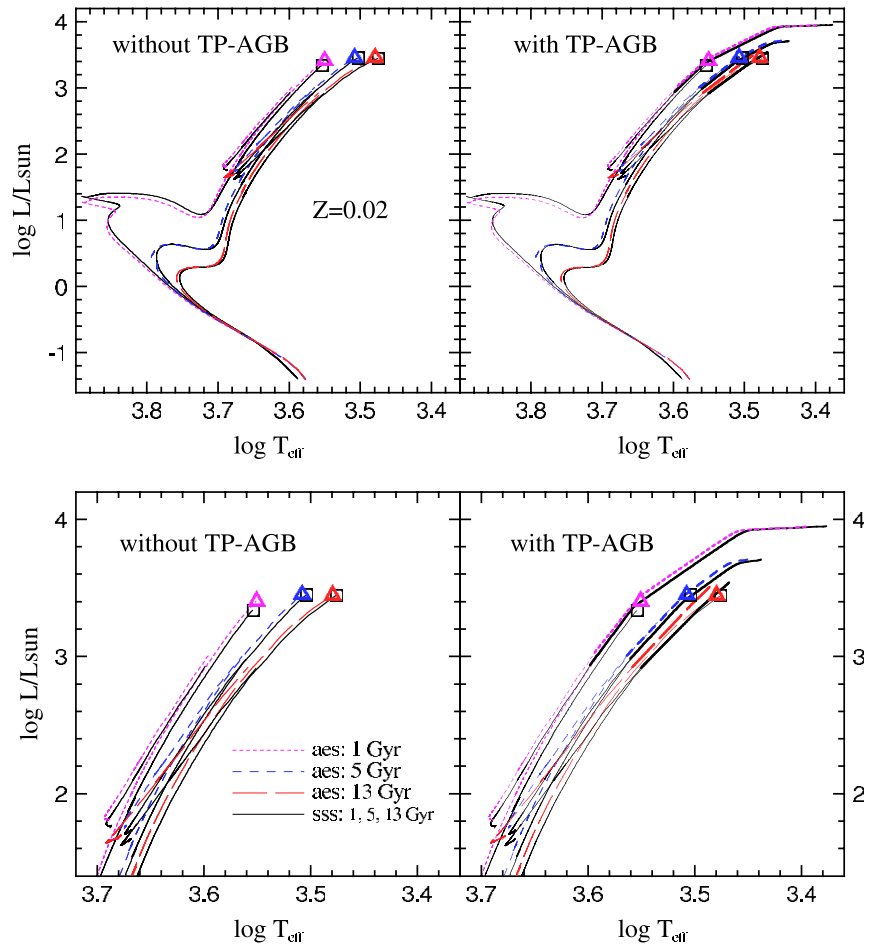

Figure 1. Comparison of solar-scaled (sss; solid lines) and $\alpha$-enhanced (aes) Teramo BaSTI isochrones in the HRD without convective core overshooting. At solar metallicity $(Z=0.02)$, three ages $(1,5,13 \mathrm{Gyr})$ are compared. Left panels display isochrones without TP-AGB, while right panels show isochrones with TP-AGBs (thicker lines). RGB tips are depicted with squares for the sss and triangles for the aes, respectively. Bottom panels show the details in the giant branches. The RGB temperatures are generally slightly warmer $(\sim 45 \mathrm{~K})$ with $\alpha$-enhancement at fixed total metallicity, partly reflecting the depression of iron abundance. Note also from the right panels that at younger ages $(t<5 \mathrm{Gyr})$, the TP-AGBs go far cooler and brighter compared to their RGB tips.

(A color version of this figure is available in the online journal.)

from stellar modelers' Web sites. It is our intention though for future study to investigate those stochastic variations.

In Section 2.2, we elaborate the nature of the $\alpha$-enhancement in stellar models, particularly the different definitions and degrees of $\alpha$-element mixture by different groups and their implications.

\subsection{HRDs and CMDs}

Here we present, for the first time, $\alpha$-element-enhanced SBF models and compare them with observations. Before we fully examine the model outputs and the comparisons with observations, however, we first look into the $\alpha$-element effects at the $\mathrm{H}-\mathrm{R}$ diagrams (HRDs) and the color-magnitude diagrams (CMDs) as well as at the emergent fluxes. The reason that we primarily employ the Teramo BaSTI Isochrones in this study is because the Teramo BaSTI stellar models provide both solarscaled and $\alpha$-enhanced isochrones with correct matching of stellar model atmospheres (Cassisi et al. 2004) all the way to the full TP-AGB stages that are crucial for the SBF calculations.

Figure 1 contrasts the solar-scaled standard (sss; by "standard," we indicate that they adopt no convective-core overshooting) Teramo BaSTI isochrones with $\sim 0.4$ dex $\alpha$-elementenhanced ones (aes) in the $\log T_{\text {eff }}$ versus $\log L / L_{\odot}$ plane. At solar metallicity $(Z=0.02)$, the Teramo BaSTI sss and aes isochrones with (right panels) and without (left panels) TPAGBs are compared at three given ages $(1,5,13 \mathrm{Gyr})$. The sss isochrones are solid lines, while the aes ones are depicted
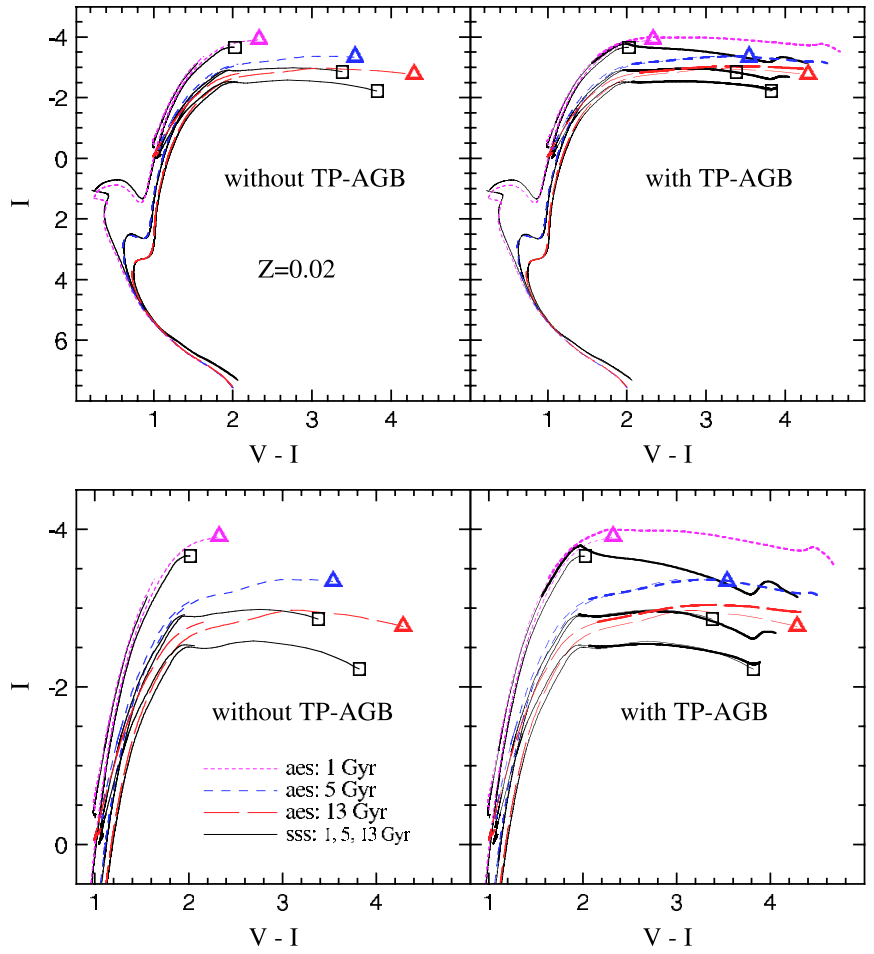

Figure 2. Similar to Figure 1, but here we display the comparison of the sss and the aes Teramo BaSTI isochrones in the $V-I$ vs. $I$ CMDs. Symbols for the RGB tips and the TP-AGBs are same as in Figure 1. Note from the left panels that the $\alpha$-enhanced RGBs, especially at the upper part $(I<-2)$, are relatively brighter and redder in this $V-I$ vs. $I$ CMDs compared to that of the solar-scaled. Similarly, it is noted from the right panels that the $\alpha$-enhanced TP-AGBs are also comparatively brighter and redder than the solar-scaled models. Moreover, it is seen from the right panels that at younger ages $(t<5 \mathrm{Gyr})$, the TP-AGBs go far redder compared to their RGB tips.

(A color version of this figure is available in the online journal.)

with dashed lines. To guide the eye, RGB tips are denoted with squares for the sss and triangles for the aes, respectively. Bottom panels show the details in the giant branches. In general, the aes isochrones are of slightly higher temperature in the RGB $(\sim 45$ $\mathrm{K})$ as well as in the upper MS compared to the solar-scaled ones. They also show slightly lower luminosity in the subgiant branch at 1 Gyr. These effects are a reflection of Fe-depression (see Figure 10 of Dotter et al. 2007a) traded with $\alpha$-element enhancement at fixed total metallicity, $Z$. The right panels of Figure 1 also show that at younger ages $(t<5$ Gyr), the TPAGBs go far cooler and brighter compared to their RGB tips (e.g., Iben \& Renzini 1983).

$V-I$ versus $I$ CMDs are displayed in Figure 2 in order to compare the sss Teramo BaSTI isochrones with the aes ones. RGB tips are again denoted with squares for the sss and triangles for the aes, respectively, to guide the eye. It is shown that the $\alpha$-enhanced RGBs, especially at the upper part $(I<-2)$, are relatively redder and brighter in this $V-I$ versus $I$ CMDs compared to the solar-scaled ones. The right panels of Figure 2 illustrate that the $\alpha$-enhanced TP-AGBs are also relatively redder and brighter in the $V-I$ versus $I$ CMDs compared to the solar-scaled ones. This is mainly because of the oxygen enhancement among $\alpha$-elements (see Figure 3). The following section addresses this issue in detail.

\subsection{Clarification of $\alpha$-element Enhancement in Stellar Models}

There are now several versions of $\alpha$-element-enhanced stellar models in the literature (e.g., Salasnich et al. 2000; Kim et al. 


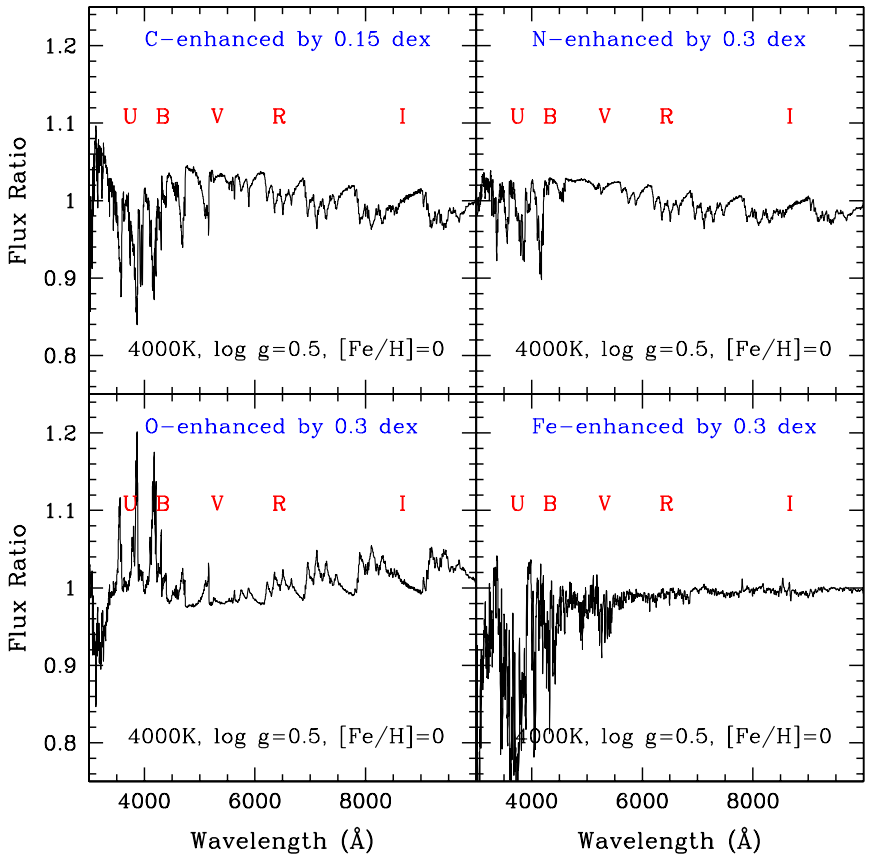

Figure 3. At $4000 \mathrm{~K}, \log g=0.5$, and $[\mathrm{Fe} / \mathrm{H}]=0$, the solar-scaled spectrum is divided by the 0.15 dex carbon-enhanced spectrum (upper left), the 0.3 dex nitrogen-enhanced spectrum (upper right), the 0.3 dex oxygen-enhanced spectrum (lower left), and the 0.3 dex iron-enhanced spectrum (lower right), respectively. $U B V R I$-band filter locations are indicated. It is seen from the lower left panel that the oxygen enhancement makes the higher $I$-band luminosity and the redder $V-I$ color, while from the upper panels it is noted that carbonand nitrogen-enhancements make the bluer $V-I$ color at this temperature and surface gravity of the typical upper RGB and AGB. From the comparison of C-, $\mathrm{N}$-, and O-enhanced cases, it is identified that many features around the $I$ band are $\mathrm{CN}$ bands and that they are more sensitive to the carbon abundance than to the nitrogen. Furthermore, it is seen from the lower right panel that many strong iron absorption line features are mostly located within the $U B V$ bandpass. Note also that the $I$-band luminosity is relatively insensitive to the iron abundance.

(A color version of this figure is available in the online journal.)

2002; Vandenberg et al. 2006; Dotter et al. 2007a, 2007b, 2008) besides the Teramo BaSTI ones of Pietrinferni et al. (2006) that we employ in this study. One should, however, carefully examine (1) whether their $\alpha$-element enhancement is defined at fixed total metallicity, $Z$ or at fixed $[\mathrm{Fe} / \mathrm{H}]$ and (2) how their $\alpha$ element mixture is defined for their $\alpha$-element-enhanced stellar models. In this study, we are contrasting the Teramo BaSTI stellar models with the Dartmouth ones (Dotter et al. 2008) and duly note that different $\alpha$-element mixtures by different groups have significant differences even though they are all commonly referred to as " $\alpha$-element-enhanced stellar models."

We have scrutinized, for example, the differences between Figure 1 of this paper and the results shown by Dotter et al. (2007a). Compared to our Figure 1, Figure 11 of Dotter et al. (2007a) shows almost no temperature changes, although they are similarly $\alpha$-element-enhanced isochrones at fixed $Z$. The culprit is the $\alpha$-element mixture. Although the Teramo BaSTI $\alpha$-enhanced stellar models are of $[\alpha / \mathrm{Fe}] \sim 0.4 \mathrm{dex}$, this is an average only. The Dartmouth models' $\alpha$-enhanced mix, in contrast, is a constant enhancement of all $\alpha$-elements with respect to solar ratios. From inspection of Table 1 of Pietrinferni et al. (2006), their oxygen abundance is very high, close to the Dartmouth models' $[\alpha / \mathrm{Fe}]=+0.8$ dex value. With this information in mind, the behavior in the HRD of Teramo BaSTI's $\alpha$-enhanced isochrones makes sense if they are compared with Figure 7 (oxygen-enhanced) in Dotter et al. (2007a). It is, in fact, the main reason why the New Standard
Stellar Population Models (NSSPM) project has opened up new windows on element-by-element variations in order to decipher the rather cryptic collection of $\alpha$-element mixtures (Dotter et al. 2007a; Lee et al. 2009).

It would be useful to compare different sets of $\alpha$-enhanced stellar population models to see if they predict the same effects of $\alpha$-enhancement on SBF. Unfortunately, we do not have the luxury of investigating several different versions of $\alpha$-enhanced SBF models at the moment. Padova isochrones and stellar evolutionary tracks are perhaps more sophisticated at the TPAGB stages compared to the Teramo BaSTI ones (e.g., Marigo et al. $2008^{5}$ ), but the Padova ones do not yet provide the matching $\alpha$-enhanced stellar evolutionary tracks and isochrones. Dotter et al. (2008; Dartmouth stellar evolutionary models), Kim et al. (2002; Yonsei-Yale models), and Vandenberg et al. (2006; Victoria-Regina models) all do provide the $\alpha$-enhanced stellar models as well as the solar-scaled ones, but they do not provide the matching TP-AGB stages that are crucial for the SBF models. These three sets are of keen interest because they present the $\alpha$-enhanced stellar models at fixed $[\mathrm{Fe} / \mathrm{H}]$ instead of at fixed $Z$ as the Teramo BaSTI stellar models do. Regarding the $\alpha$-enhancement at fixed $Z$, a depressed $\mathrm{Fe}$ abundance preserves the total metallicity. For instance, in the Teramo BaSTI stellar models, there is about $0.35 \mathrm{dex}[\mathrm{Fe} / \mathrm{H}]$ shift at fixed total metallicity, $Z$, between the solar-scaled (sss; solid lines) and the $\alpha$-enhanced (aes; dashed lines) models. The case of fixed $[\mathrm{Fe} / \mathrm{H}]$ is, alas, also less than perfectly straightforward. In that case, the enhancement of the $\alpha$-elements increases the overall metallicity, and therefore either the abundance of hydrogen or helium (or both) must be modified in order to compensate for the increased $Z$.

Moreover, there is the stellar atmosphere (emergent flux) issue. The Teramo BaSTI $\alpha$-enhanced stellar models incorporate the matching $\alpha$-enhanced stellar model atmospheres selfconsistently in order to generate the observables (magnitudes and colors) as described in Cassisi et al. (2004). All the other $\alpha$-enhanced stellar models, however, employ the solar-scaled stellar atmosphere for their calculations of $\alpha$-enhanced model observables. It would be useful to have $\alpha$-enhanced models at fixed $[\mathrm{Fe} / \mathrm{H}]$ from the Teramo BaSTI group, as well as at fixed $Z$, so that the effects of the $\alpha$-element variation could be seen more directly, instead of mixing in the effects of Fe depression for the $\alpha$-enhanced models at fixed $Z$ (Salaris et al. 1993). In the same context, we are also looking forward to implementing the Padova $\alpha$-enhanced stellar models with matching $\alpha$-enhanced stellar model atmospheres as they become available.

Figure 3 demonstrates how the enhancement of each element (carbon, nitrogen, oxygen, and iron) modifies the emergent fluxes at $4000 \mathrm{~K}$ and $\log g=0.5$, which is the typical temperature and surface gravity of the upper RGBs and AGBs. The lower left panel of Figure 3 illuminates that the oxygen enhancement generates a brighter $I$-band luminosity and a redder $V-I$ color. From Table 1 , it is noted that the $V-I$ color becomes 0.031 mag redder because of the 0.3 dex oxygen enhancement. Being a dominant $\alpha$-element (e.g., $\mathrm{O}, \mathrm{Mg}, \mathrm{Si}, \mathrm{S}, \mathrm{Ca}, \mathrm{Ti}$ ), this behavior from the oxygen-enhanced spectrum is very useful in order to understand the $V-I$ colors and $I$-band magnitudes seen in Figure 2 as well as the model results in Figure 4 that

\footnotetext{
5 Table 1 of Marigo et al. (2008) lists the available stellar isochrones including the TP-AGB phase. Marigo et al. (2008) note that the TP-AGB of the Teramo BaSTI isochrones by Cordier et al. (2007) was computed in a rather crude way, i.e., without considering the third dredge-up events (carbon star formation) and hot bottom burning nucleosynthesis.
} 


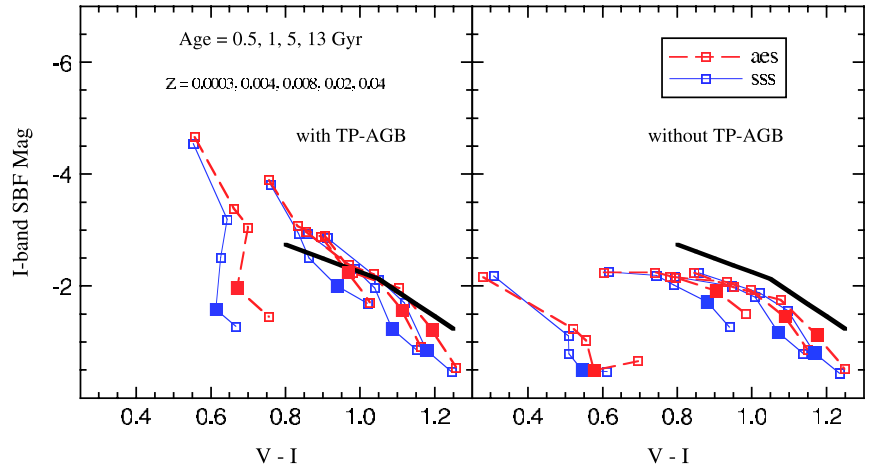

Figure 4. $I$-band SBF magnitudes as a function of integrated $V-I$ colors are shown at $0.5,1,5$, and $13 \mathrm{Gyr}$ (left to right) for five different metallicities employing the most up-to-date Teramo BaSTI isochrones available after 2008 May. Solid lines with squares are solar-scaled models, while dashed lines with squares are $\alpha$-enhanced models. At given ages, $I$-band SBF magnitudes become fainter and $V-I$ colors become redder, in general, with increasing metallicity. To guide the eye, solar metallicity $(Z=0.02)$ is depicted with filled squares. Two observational fiducial lines (bent thick solid lines; bluer one from Mieske et al 2006 and redder one from Tonry et al. 2000) are compared with our theoretical models. The $\alpha$-enhanced models at solar metallicity, $Z=0.02$ (filled squares), become redder and brighter (please see Table 3 for the values) compared to the solar-scaled ones mostly because of the oxygen enhancement as we noted in Figures 2 and 3. The models without TP-AGBs in the right panel show that the general effect of the $\alpha$-enhanced models at solar metallicity $(Z=0.02$, filled squares) becoming redder and brighter compared to the solar-scaled ones for $t \geqslant 1$ Gyr are mostly unchanged. It is noted, however, that the $I$-band SBF magnitudes are much too faint without TP-AGBs to match the observations.

(A color version of this figure is available in the online journal.)

Table 1

Color Behavior with Elemental Enhancements for a Star with $T_{\text {eff }}=4000 \mathrm{~K}$, $\log g=0.5$

\begin{tabular}{lcrrrrrrrr}
\hline \hline Color & $\mathrm{C}$ & $\mathrm{N}$ & $\mathrm{O}$ & $\mathrm{Mg}$ & $\mathrm{Si}$ & $\mathrm{S}$ & $\mathrm{Ca}$ & $\mathrm{Ti}$ & $\mathrm{Fe}$ \\
\hline$U-B$ & 30 & 26 & -21 & 34 & -43 & 0 & 16 & 24 & 84 \\
$B-V$ & 16 & -2 & -13 & -35 & 18 & 0 & 7 & 10 & 13 \\
$V-I$ & -30 & -28 & 31 & 12 & 0 & 0 & -4 & 2 & 16 \\
\hline
\end{tabular}

Notes. (1) All elements scaled individually by +0.3 dex, except $\mathrm{C}$, which is increased by +0.15 dex. (2) Numbers are in milli-magnitude.

we discuss in the following section. The upper panels illustrate the carbon- and nitrogen-enhanced spectra again at $4000 \mathrm{~K}$ and $\log g=0.5$. They are, in general, displaying the opposite from what we see in the lower left panel of Figure 3. By comparing the $\mathrm{C}-, \mathrm{N}$-, and O-enhanced spectra, it is interesting to find that many features around the $I$ band are $\mathrm{CN}$ bands, and they are more sensitive to carbon abundance than to nitrogen. The oxygenenhanced spectrum in the lower left panel of Figure 3 illustrates that increasing oxygen abundance soaks up more $\mathrm{C}$ into the $\mathrm{CO}$ molecule, decreasing $\mathrm{C}_{2}, \mathrm{CH}$, and $\mathrm{CN}$ feature strengths.

The lower right panel of Figure 3 shows the iron-enhanced spectrum at $4000 \mathrm{~K}$ and $\log g=0.5$. It is evident that many strong iron absorption line features occur around the $U B V$ bandpasses. From Table 1 , it is noted that the $U-B$ color becomes 0.084 mag redder because of the 0.3 dex iron enhancement. Moreover, it is worth emphasizing that $I$-band luminosity is relatively insensitive to the iron abundance. The lower right panel of Figure 3 is quite helpful in understanding the observed color-magnitude effects because the $\alpha$-enhanced Teramo BaSTI stellar models are essentially equivalent to $\mathrm{Fe}$ depressed ones.

\section{RESULTS}

Having discussed the nature of the $\alpha$-element enhancement in terms of the isochrones and the emergent fluxes, we now present the integrated $\alpha$-enhanced SBF models and broadband colors. Following our initial results on this topic (Lee et al. 2007a), there have been critical updates from the Teramo BaSTI stellar models lately. The $\alpha$-enhanced isochrones and stellar evolutionary tracks have been recomputed for $Z>0.001$ after employing the low-temperature opacities by Ferguson et al. (2005). ${ }^{6}$

Figure 4 shows our new I-band SBF model calculations (aes: $\alpha$-enhanced standard, sss: solar-scaled standard, where "standard" again means no convective core overshooting) as a function of integrated $V-I$ colors based upon the recently updated (after 2008 May) Teramo BaSTI isochrones. Two observational fiducial lines (thick bent straight lines) are overlaid with our theoretical models. The line on the blueside is $I$-band $\mathrm{SBF}$ Mag $=-2.25+2.44 \times[(V-I)-1.00]$ from Mieske et al. $(2006)^{7}$ for dwarf blue galaxies, while the line on the redside is $I$-band SBF Mag $=-1.68+4.5 \times[(V-I)-1.15]$ from Tonry et al. $(2000)^{8}$ for massive red galaxies. It is worth mentioning here that we use the observational fiducial lines merely as a "sanity check." As we mentioned in Section 2, we do not consider the stochastic nature of the TP-AGB in this study. Therefore, we want to make it clear that our aim in this study is the blunt investigation of the effects of $\alpha$-element enhancement and the TP-AGB stars on the SBF magnitudes and broadband colors of simple stellar populations.

Compared to our earlier results (Lee et al. 2007a), the differences between the solar-scaled and the $\alpha$-enhanced models are smaller mainly because of the Teramo BaSTI isochrones updates (due to the low temperature opacities by Ferguson et al. 2005 for the $\alpha$-enhanced stellar models). At solar metallicity $(Z=0.02$, filled squares), it is found from Figure 4 (also from Table 3 ) that the $\alpha$-enhanced models become about 0.02 mag redder and 0.35 mag brighter in this integrated $V-I$ versus $I$-band SBF magnitude plane compared to the solar-scaled ones mostly because of the oxygen enhancement as we noted in Figures 2 and 3. The right panel of Figure 4 displays the integrated $V-I$ colors versus $I$-band SBF magnitudes when the TP-AGBs are not included in the calculations. The general trend of the $\alpha$-enhanced models at solar metallicity $(Z=0.02$, filled squares) becoming redder and brighter compared to the solar-scaled ones for $t \geqslant 1 \mathrm{Gyr}$ is mostly intact. It is seen, however, that the I-band SBF magnitudes are much too faint without the TP-AGBs to match the observations, especially at the metal-poor end.

Figure 5 is similar to Figure 2, but here displays the comparison of the sss and the aes Teramo BaSTI isochrones in $V-I$ versus $I$ CMDs at $Z=0.0003$. It is important to note here that compared to Figure 2, the $V-I$ colors and the $I$-band magnitudes are hardly changed with the $\alpha$-enhancement on this very metal-poor side even at the upper RGB. It explains the comparably smaller effects of $\alpha$-enhancement on SBF models and

\footnotetext{
6 See "News" in 3/30/2007 and 5/16/2008 at http://193.204.1.62/index.html for the details.

7 In Mieske et al. (2006), $I$-band SBF Mag $=-2.13( \pm 0.17)+2.44( \pm 1.94)$ $\times[(V-I)-1.00]$ for $0.8<V-I<1.10$. We have shifted within the permitted errors in order to match with the empirical line by Tonry et al. (2000).

8 We have adopted 0.06 mag zero-point correction by Blakeslee et al. (2002) to match the final set of $H_{0}$ Key Project Cepheid distances from Freedman et al. (2001).
} 


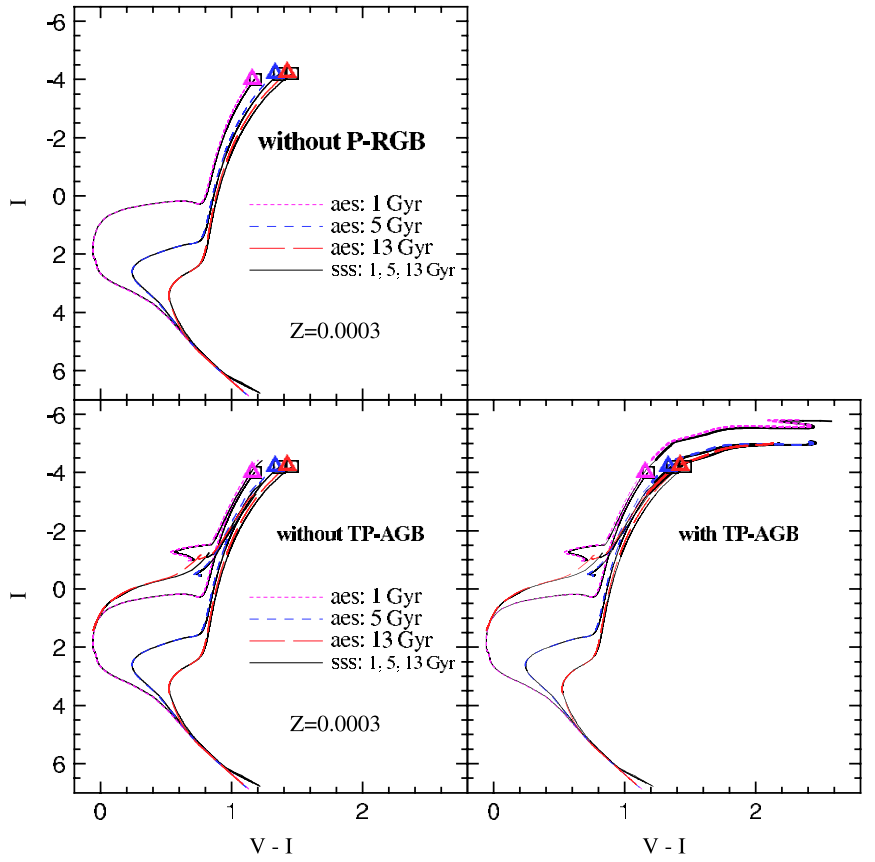

Figure 5. Similar to Figure 2, but here we display the comparison of the solarscaled (sss) and the $\alpha$-enhanced (aes) Teramo BaSTI isochrones in the $V-I$ vs. $I \mathrm{CMD}$ at $Z=0.0003$. Symbols for the RGB tips and the TP-AGBs are same as in Figure 1. Top left panel shows the CMD without post-RGB (p-RGB) stars. From left panels, it is interesting to find that the blue horizontal branch at $13 \mathrm{Gyr}$ overlaps in $V-I$ color and $I$-band magnitude with the MS turnoff of $1 \mathrm{Gyr}$ here at $Z=0.0003$. Note that compared to Figure 2, the $V-I$ colors and the $I$-band magnitudes are hardly changed with the $\alpha$-enhancement at this very metal-poor regime even at the upper RGB. It explains the comparably smaller effects of $\alpha$-enhancement at $Z=0.0003$ in Figure 4. Also, note from the right panel that at all ages, the TP-AGBs go far redder and brighter compared to their RGB tips, particularly at this very metal-poor regime, which explains the importance of the TP-AGBs at $Z=0.0003$ shown in Figure 4 .

(A color version of this figure is available in the online journal.)

integrated broadband colors at $Z=0.0003$ in Figure 4. It is also interesting to see from the left panels (upper: without postRGB; lower: with post-RGB but without TP-AGB) that the blue horizontal branch of $13 \mathrm{Gyr}$ overlaps in $V-I$ color and $I$-band magnitude with the MS turnoff of 1 Gyr here at $Z=0.0003$. In the right panel of Figure 5, TP-AGBs are additionally depicted with thicker lines. It is seen that the TP-AGBs are more than 1 mag redder in $V-I$ and 1-2 mag brighter in $I$-band magnitude compared to their RGB tips, particularly on this very metal-poor side (compare, right panels of Figure 2). It explains the importance of the TP-AGBs on SBF models and integrated broadband colors at $Z=0.0003$ in Figure 4 (see also Figure 14).

We also address the convective core overshooting issue at young ages $(t<5 \mathrm{Gyr})$. Figure 6 is similar to the left panel of Figure 4 , but here integrated $V-I$ colors and $I$-band SBF magnitudes using the Teramo BaSTI aes (dashed lines; $\alpha$ enhanced and without convective core overshooting) and aeo (solid lines; $\alpha$-enhanced and with convective core overshooting) isochrones are compared at three different relatively young ages. Note that at $1 \mathrm{Gyr}$ and solar metallicity (filled squares) overshooting effects make the integrated $V-I$ colors $0.05 \mathrm{mag}$ bluer and the $I$-band SBF magnitudes $0.18 \mathrm{mag}$ fainter as indicated with an arrow (see also Table 3). At 5 Gyr, however, it is seen that the overshooting effects become negligible. Figure 7 is similar to the right panels of Figures 1 and 2, but the aes (dashed lines) and the aeo (solid lines) Teramo BaSTI isochrones are compared in the HRDs and the CMDs at 1 and $5 \mathrm{Gyr}$ for

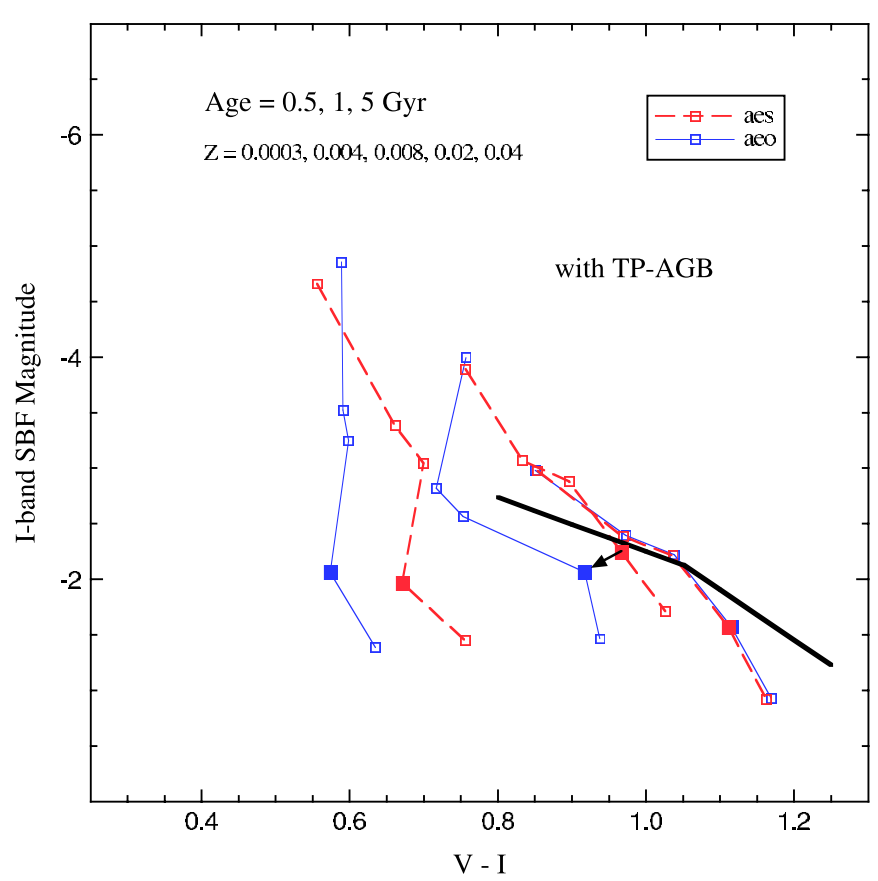

Figure 6. Similar to the left panel of Figure 4, but here $\alpha$-enhanced $I$-band SBF magnitudes as a function of $V-I$ are compared employing the Teramo BaSTI aes (dashed lines; without convective core overshooting) and aeo (solid lines; with convective core overshooting) isochrones at three given ages. The observational fiducial lines are same as in Figure 4. Note that at $1 \mathrm{Gyr}$ and solar metallicity ( $Z=0.02$, filled squares), overshooting effects make the integrated $V-I$ colors bluer and the $I$-band SBF magnitudes fainter as indicated with an arrow. At 5 Gyr, however, the overshooting effects become minimal.

(A color version of this figure is available in the online journal.)

$Z=0.02$. The RGB tips are denoted with triangles for the aes and circles for the aeo, respectively, to guide the eye. It is interesting to note that the aeo models (1) have a hotter upper MS near the turnoff compared to the aes ones and (2) do not go all the way to the RGB tip at $1 \mathrm{Gyr}$ (see also Lee et al. 2007b; Yi 2003). However, the aes and aeo models become virtually identical by $5 \mathrm{Gyr}$. The behavior due to the overshooting effects on the integrated $V-I$ colors (bluer because of the bluer upper MS near the turnoff and the fainter RGB) and on the $I$-band SBF magnitudes (fainter because of the less developed upper RGB) that we described in Figure 6 can be understood from this CMD.

\subsection{Comparison with Earlier Models}

Figures 8 and 9 compare our scaled-solar (sss) models with other recent models available in the literature. Figure 8 contrasts our models based on the Teramo BaSTI "sss" isochrones with Raimondo et al. (2005), while Figure 9 does that with MarínFranch \& Aparicio (2006). ${ }^{9}$ The large filled symbols are used to indicate the solar metallicity in order to guide the eye. Both Figures 8 and 9 demonstrate that there are significant differences among models mostly because of the different treatment of the TP-AGB stars. Hence, we have collected several other available models in the literature and listed them in Table 2 . We have only listed their integrated $V-I$ colors and $I$-band SBF magnitudes at 5 and 13 Gyr at solar metallicity.

Table 2 tells that there are non-negligible disparities among models at the same age and metallicity. The two different Worthey (1994) models ${ }^{10}$ evidently show that the different input

\footnotetext{
9 Among three different models in Marín-Franch \& Aparicio (2006), we show the one with Pietrinferni et al. (2004) isochrones.

10 http://astro.wsu.edu/worthey/dial/dial_a_model.html
} 

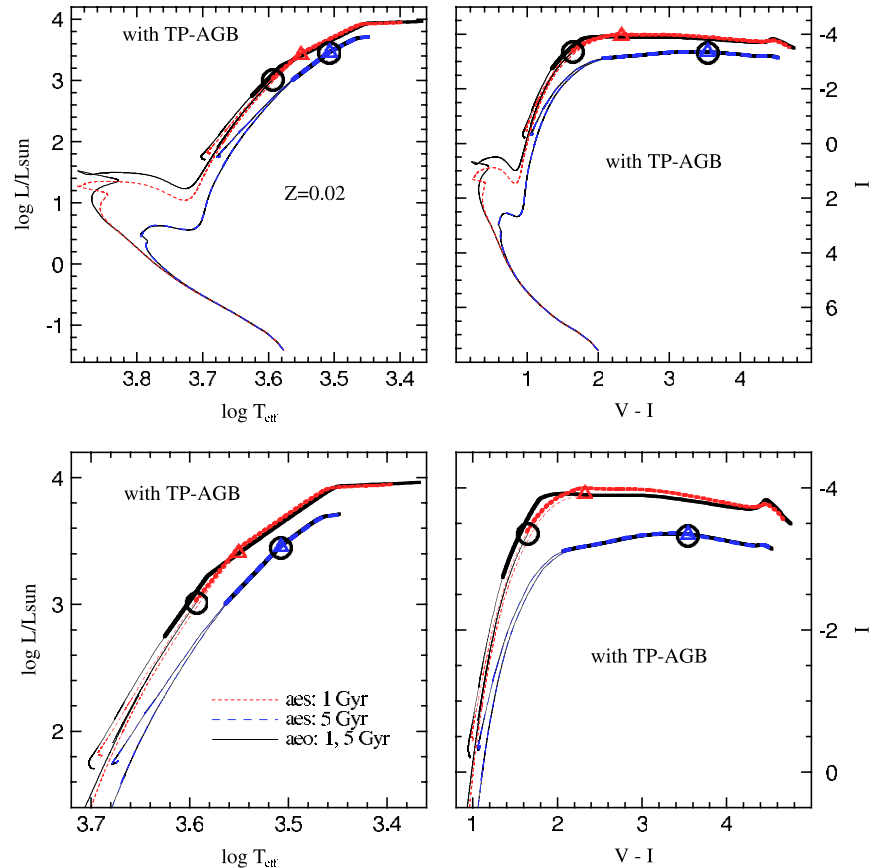

Figure 7. Similar to the right panels of Figures 1 and 2, but the aes (dashed lines; without convective core overshooting) and the aeo (solid lines; with convective core overshooting) Teramo BaSTI isochrones are compared in the HRDs (left panels) and CMDs (right panels) at 1 and $5 \mathrm{Gyr}$ at $Z=0.02$. RGB tips are denoted with triangles for the aes and circles for the aeo, respectively. Bottom panels show the details in the giant branches. It is interesting to note that the aeo models (1) are hotter at the upper MS compared to the aes models and (2) do not go all the way to the RGB tip at $1 \mathrm{Gyr}$. They become virtually identical at $5 \mathrm{Gyr}$ The overshooting effects on the integrated $V-I$ colors and the $I$-band SBF magnitudes that we described in Figure 6 can be understood from this CMD (see the text).

(A color version of this figure is available in the online journal.)

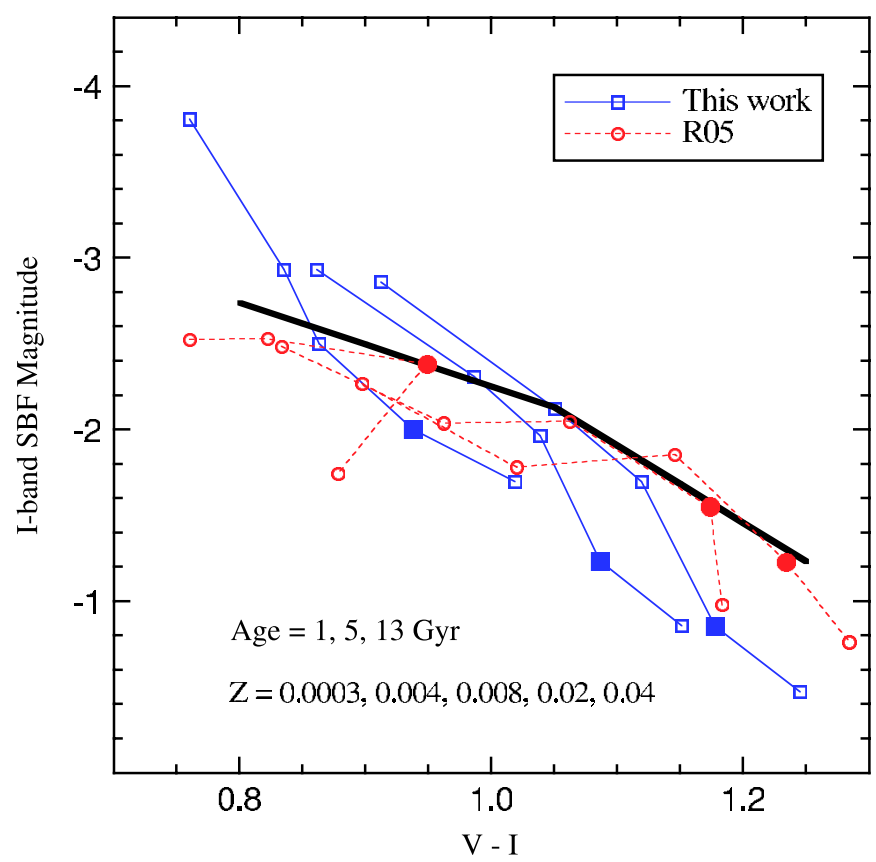

Figure 8. Similar to the left panel of Figure 4, but here our solar-scaled (sss; without convective core overshooting) $I$-band SBF models as a function of $V-I$ are compared with the Teramo SPoT models (Raimondo et al. 2005, hereafter $\mathrm{R} 05)$ at 1,5 , and $13 \mathrm{Gyr}$ for five different metallicities. To guide the eye, solar metallicity $(Z=0.02)$ is depicted with filled symbols.

(A color version of this figure is available in the online journal.)

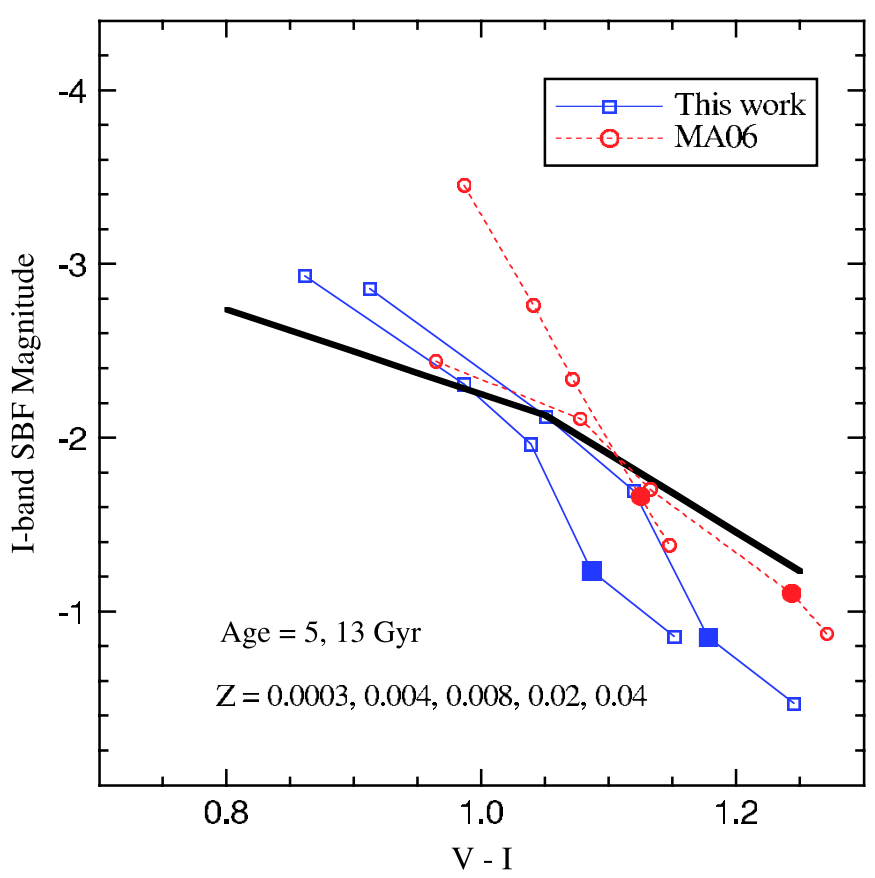

Figure 9. Similar to Figure 8, but here our solar-scaled $I$-band SBF models as a function of $V-I$ are compared from that of Marín-Franch \& Aparicio (2006, hereafter MA06) at 5, and $13 \mathrm{Gyr}$ for five different metallicities. To guide the eye, solar metallicity $(Z=0.02)$ is depicted with filled symbols.

(A color version of this figure is available in the online journal.)

ingredients (i.e., isochrones) make significant $(\sim 0.1 \mathrm{mag}$ in $V-I$ and $\sim 0.55 \mathrm{mag}$ in $I$-band SBF magnitudes) disparities at the same age and metallicity. Obviously, depending upon the ingredients and recipe the modelers adopt such as (1) isochrones, particularly the late-evolutionary stage evolutionary tracks such as RGB, AGB, and TP-AGB, (2) mass-loss scheme such as $\eta$ in Reimers (1975), (3) stellar library to convert the temperature and luminosity to colors and magnitudes, and (4) the IMF, one can get significantly different model outputs. It is therefore considerably important to carefully study the ingredients and recipe of different models before use. A rigorous test of the integrated photometric models is imperative.

On a positive note, however, our models agree extremely well with other very recent works using independent stellar population models. For example, our scaled-solar (sss) models and Percival et al. (2009, hereafter P09) that are based upon the most up-to-date Teramo BaSTI with $\eta=0.4$ and Salpeter IMF agree each other within $0.003 \mathrm{mag}$ in $V-I$. Moreover, our scaled-solar models that are based upon the most up-todate Padova isochrones with Salpeter IMF and Padova SSP models ${ }^{11}$ (Marigo et al. 2008) agree each other within $0.002 \mathrm{mag}$ in $V-I$ at solar metallicity at 13 Gyr. Our models based upon the different isochrones will be compared with one another in detail in the following section.

\subsection{Comparison of Padova and Teramo/BaSTI Solar-scaled SBF Models}

Having discovered the non-negligible impacts of the input ingredients (i.e., isochrones) on the SBF magnitudes and broadband colors, here we calculate those quantities by employing stellar models from different groups but at the same solar-scaled

\footnotetext{
11 They are available from http://stev.oapd.inaf.it/cgi-bin/cmd.
} 
Table 2

Integrated $V-I$ and $I$-band SBF Mag from Different SSP Models

\begin{tabular}{lcccc}
\hline \hline \multicolumn{1}{c}{ Models } & $V-I(5 \mathrm{Gyr})$ & $V-I(13 \mathrm{Gyr})$ & $\bar{M}_{I}(5 \mathrm{Gyr})$ & $\bar{M}_{I}(13 \mathrm{Gyr})$ \\
\hline W94, Sal & 1.186 & 1.312 & -1.680 & -1.255 \\
W94, Sal, B94 & 1.148 & 1.210 & -1.129 & -0.699 \\
B01, Sal, G00 & 1.140 & 1.240 & -1.410 & -0.960 \\
BC03, Cha, B94 & 1.124 & 1.227 & $\ldots$ & $\ldots$ \\
BC03, Sal, B94 & 1.141 & 1.255 & $\ldots$ & $\ldots$ \\
M05, Kro & 1.088 & 1.176 & $\ldots$ & $\ldots$ \\
M05, Sal & 1.099 & 1.198 & $\ldots$ & $\ldots$ \\
R05, Sca, P04 & 1.174 & 1.234 & -1.548 & -1.225 \\
MA06, Kro, B94 & 1.167 & 1.282 & -1.097 & -0.664 \\
MA06, Kro, G00 & 1.163 & 1.278 & -1.290 & -0.909 \\
MA06, Kro, P04 & 1.124 & 1.243 & -1.663 & -1.104 \\
Pad_SSP, Cha & 1.162 & 1.238 & $\ldots$ & $\ldots$ \\
Pad_SSP, Sal & 1.172 & 1.261 & $\ldots$ & $\ldots$ \\
P09, sss, 0.2 & 1.092 & 1.185 & $\ldots$ & $\ldots$ \\
P09, sss, 0.4 & 1.089 & 1.177 & $\ldots$ & $\ldots$ \\
P09, aes, 0.2 & 1.121 & 1.203 & $\ldots$ & $\ldots$ \\
P09, aes, 0.4 & 1.117 & 1.193 & $\ldots$ & $\ldots$ \\
This Work, sss & 1.086 & 1.178 & -1.235 & -0.853 \\
This Work, aes & 1.111 & 1.192 & -1.567 & -1.217 \\
This Work, Pad & 1.173 & 1.263 & -1.373 & -0.831 \\
\hline
\end{tabular}

Notes. (1) W94 (Worthey 1994) is from http://astro.wsu.edu/worthey/dial/ dial_a_model.html. "Sal" is Salpeter IMF and "B94" is Bertelli et al. (1994). (2) B01 (Blakeslee et al. 2001) are 5 and $12.6 \mathrm{Gyr}$ values from their Table 2. (3) BC03 (Bruzual \& Charlot 2003) is from http://www2.iap.fr/users/charlot/ bc2003/. "Cha" is Chabrier (2003) IMF. (4) M05 (Maraston 2005) is from http://www.icg.port.ac.uk/ maraston/SSPn/colors/SSPcolours_Mar05_ JohnsonCousins.tab. "Kro" is Kroupa (2001) IMF. (5) R05 (Raimondo et al. 2005) is from http://193.204.1.79:21075/models.html. "Sca" is Scalo (1998) IMF and "P04" is Pietrinferni et al. (2004). (6) MA06 (Marín-Franch \& Aparicio 2006) used Kroupa IMF. (7) Pad_SSP (Marigo et al. 2008) is from http://stev.oapd.inaf.it/cgi-bin/cmd. (8) P09 (Percival et al. 2009) is from http://193.204.1.62/index.html; "sss" is scaled-solar and "aes" is $\alpha$-enhanced. Two mass-loss schemes are used; one with $\eta=0.2$ and the other with $\eta=0.4$. (9) This work; sss and aes adopt the $\eta=0.4$. This work; Pad is the SSP based on Marigo et al. (2008).

composition. In this study, we contrast the widely used two stellar models, the Padova ${ }^{12}$ and the Teramo BaSTI stellar models ${ }^{13}$ at the same solar-scaled chemical composition. It is worth reiterating that $\alpha$-enhanced stellar models could be even more diverse than expected because of differing definitions of " $\alpha$-element."

Figure 10 is similar to Figure 4, but here we contrast the $I$-band SBF models as a function of integrated $V-I$ colors at given ages and metallicities using two different stellar models but at the same solar-scaled chemical composition with convective core overshooting. One is using the Teramo BaSTI (solid lines), and the other is using the Padova (dashed lines) stellar models. We employ their latest stellar models, which we directly download from their Web sites (see footnotes 1 and 9, respectively) in order to calculate the SBF predictions. We have employed the "sso" (solar-scaled with convective core overshooting) Teramo BaSTI models because we note that the Padova stellar models employ the convective core overshoot as the default in their models. ${ }^{14}$ To guide the eye, solar metallicity models of varying ages are marked with filled squares. The two sets of stellar models result in significant disparities. In general, integrated $V-I$ colors based on the Padova isochrones are comparatively redder than

\footnotetext{
12 http://stev.oapd.inaf.it/cgi-bin/cmd

13 http://193.204.1.62/index.html

14 Please refer to footnotes 1 and 9 for the detailed treatment of the convective core overshooting from each stellar model group.
}

Table 3

SBF Magnitude and Integrated $V-I$ Color Differences at $Z=0.02$

\begin{tabular}{|c|c|c|c|}
\hline $\begin{array}{l}\text { Age } \\
\text { (Gyr) }\end{array}$ & $\begin{array}{c}\Delta I \text {-band SBF } \\
(\mathrm{mag})\end{array}$ & $\begin{array}{c}\Delta F 160 W \text {-band SBF } \\
(\mathrm{mag})\end{array}$ & $\begin{array}{c}\Delta V-I \\
(\mathrm{mag})\end{array}$ \\
\hline \multicolumn{4}{|c|}{ Effects of 0.4 dex $\alpha$-enhancement using Teramo BaSTI: aes - sss } \\
\hline 0.5 & -0.380 & 0.168 & 0.058 \\
\hline 1 & -0.242 & 0.152 & 0.029 \\
\hline 5 & -0.332 & 0.080 & 0.025 \\
\hline 13 & -0.364 & 0.066 & 0.014 \\
\hline
\end{tabular}

\begin{tabular}{lccc} 
& \multicolumn{3}{c}{ TP-AGB } \\
0.5 & -1.084 & -4.571 & 0.068 \\
1 & -0.286 & -1.880 & 0.057 \\
5 & -0.068 & -0.472 & 0.015 \\
13 & -0.046 & -0.207 & 0.011 \\
& Effects of convective core overshooting (using Teramo BaSTI): aeo - aes \\
0.5 & -0.094 & $\ldots$ & -0.098 \\
1 & 0.180 & $\ldots$ & -0.050 \\
5 & -0.003 & $\ldots$ & 0.004
\end{tabular}

BaSTI

\begin{tabular}{lrrr}
0.5 & -1.444 & -0.736 & 0.041 \\
1 & -0.743 & -0.945 & -0.036 \\
5 & -0.138 & -0.546 & 0.087 \\
13 & 0.022 & 0.006 & 0.085 \\
\hline
\end{tabular}

Notes. (1) The negative numbers in Columns 2 and 3 indicate that the effects make the SBF magnitude brighter and vice versa. (2) The positive numbers in Column 4 indicate that the effects make the integrated $V-I$ color redder and vice versa.

that from the Teramo BaSTI (see Table 3). It is also noted from the left panel that the $I$-band SBF magnitudes based upon the Teramo BaSTI become much fainter $(>1 \mathrm{mag})$ at younger ages $(t<5$ Gyr) with $Z \geqslant 0.0004$ compared to Padova models. In order to ascertain whether the remarkable dissimilarities are mainly caused by the rather poorly understood bright TP-AGB stars, we display the same models without TP-AGBs in the right panel of Figure 10. The differences using two different stellar models persist even without the TP-AGBs, although they are much less compared to that with the TP-AGBs.

From Figure 10, however, it is evident that the inclusion of TPAGB stages is indeed necessary in matching the observations, which are represented by the thick bent lines. The systematic redder integrated $V-I$ colors using the Padova stellar models compared to that using the Teramo BaSTI ones can be understood from the fact that the Padova RGBs are systematically cooler and redder than the Teramo BaSTI ones, as illustrated in Figures 11 and 12. For a clearer understanding of the cause of the dissimilarities of the stellar population model $I$-band SBF predictions as well as the integrated $V-I$ colors using the Padova and the Teramo BaSTI stellar models, we illustrate the comparison of the Padova and the Teramo BaSTI stellar models in the HRDs and the CMDs in the following figures.

Figure 11 shows the comparison of the Teramo BaSTI (solid lines) and the Padova (dashed lines) isochrones at the same solar-scaled chemical composition in the HRDs without (left panels) and with (right panels) TP-AGB stages, respectively. At solar metallicity, three ages $(1,5,13 \mathrm{Gyr})$ are compared. RGB tips are denoted with squares for Teramo BaSTI and triangles for Padova, respectively. From Figure 11, it is noted that the main-sequence turn off and RGB temperatures of the older ages $(t \geqslant 5 \mathrm{Gyr})$ are generally cooler in the Padova stellar models, which cause the redder integrated $V-I$ colors compared to those using the Teramo BaSTI ones in Figure 10. 


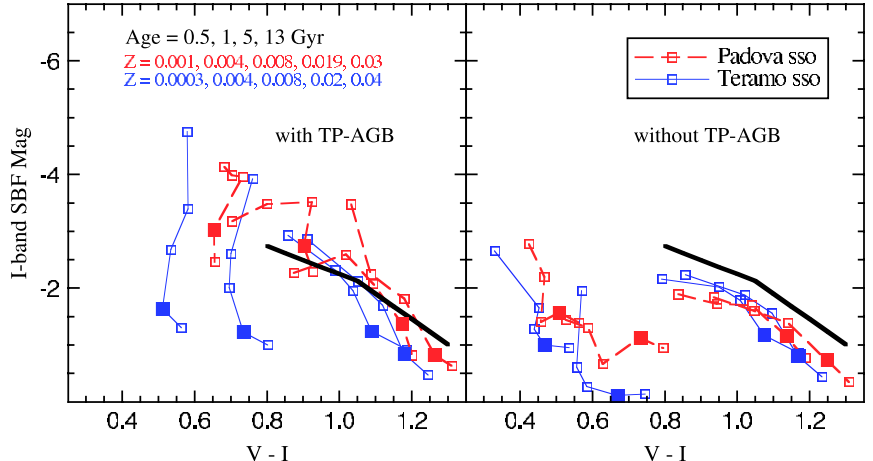

Figure 10. Similar to Figure 4, but here we contrast the $I$-band SBF models as a function of integrated $V-I$ colors at the same solar-scaled compositions (sso; convective core overshooting is "on") using the Padova (dashed lines) and the Teramo BaSTI (solid lines) stellar models at given ages and metallicities. To guide the eye, solar metallicity is depicted with filled squares. The observational fiducial lines are same as in Figure 4. In general, $V-I$ colors employing the Padova isochrones are comparatively redder than that employing the Teramo BaSTI. It is noted from the left panel that the $I$-band SBF magnitudes based upon the Teramo BaSTI are more than 1 mag fainter at younger ages $(t<5 \mathrm{Gyr})$ with $Z>0.004$ compared to that employing Padova stellar models. The model disparities based on the two different stellar models linger even without TPAGBs as shown in the right panel. It is evident though that the inclusion of TP-AGB stages is crucial to match the observations, which are the bent thick lines.

(A color version of this figure is available in the online journal.)
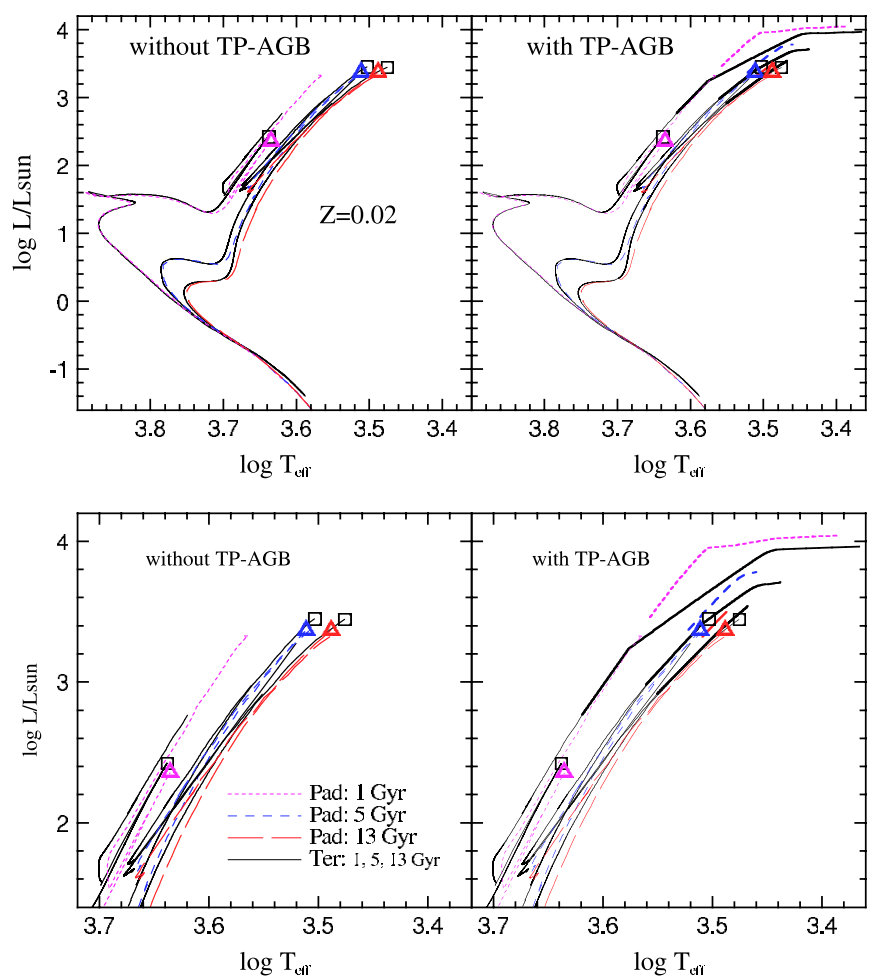

Figure 11. Comparison of the Teramo BaSTI (solid lines) and the Padova (dashed lines) isochrones at the same solar-scaled composition (sso; convective core overshooting is "on") in the HRDs without (left panels) and with (right panels) TP-AGB stages. At solar metallicity, three ages (1, 5, and 13 Gyr) are compared. RGB tips are marked with squares for Teramo BaSTI and triangles for Padova, respectively. Bottom panels show the giant branches in details. Note that the RGB temperatures from the Padova stellar models are generally cooler than that from the Teramo BaSTI models and they become redder $V-I$ colors as shown in Figure 12.

(A color version of this figure is available in the online journal.)

The small RGB temperature differences may be ascribed to the uncertainties of the convection treatment in the RGB stars. From
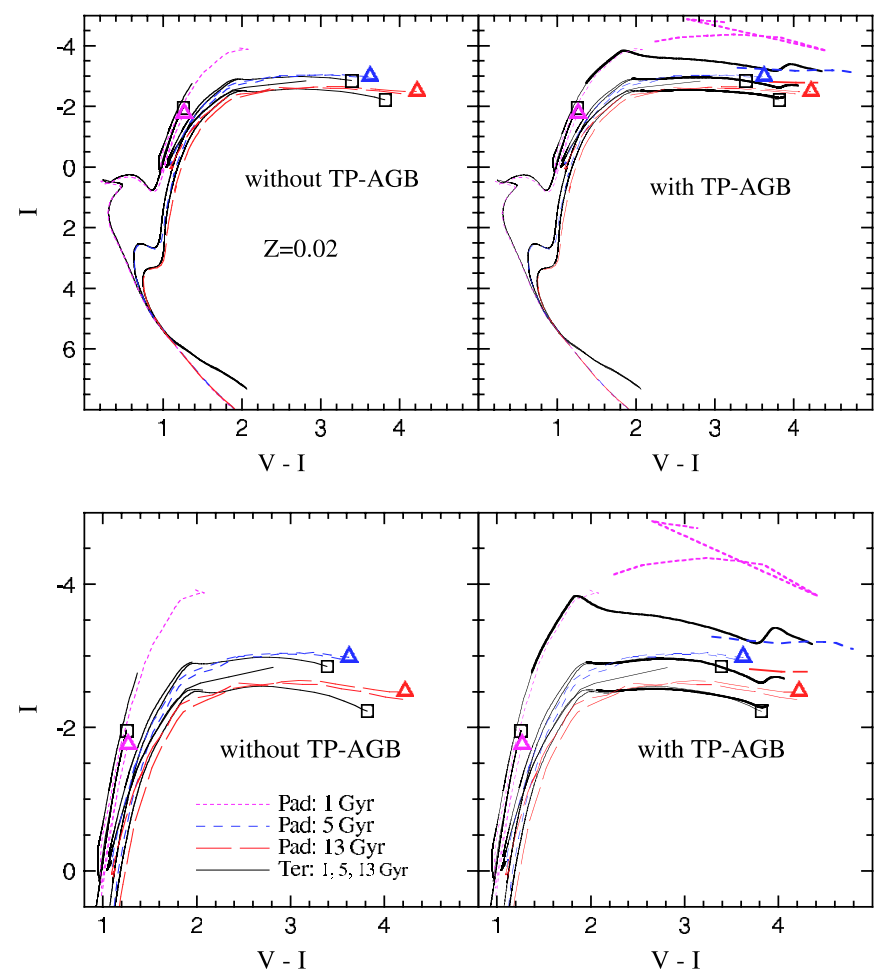

Figure 12. Similar to Figure 11, but here displaying the comparison of the Teramo BaSTI (solid lines) and the Padova (dashed lines) isochrones in $V-I$ vs. ICMDs. Symbols for the RGB tips are same as in Figure 11. Bottom panels show the giant branches in details. Note that the Padova giant branches, especially at the upper part, are relatively redder than the Teramo BaSTI ones in this $V-I$ vs. $I$ CMDs. Note also from the right panels that there are some discontinuities for the Padova stellar models at the onset of TP-AGBs because of their structural changes (see the text).

(A color version of this figure is available in the online journal.)

the right panels of Figure 11, it is also noted that at younger ages $(t<5$ Gyr), the TP-AGBs go far cooler and brighter than their RGB tips.

Figure 12 displays the comparison of the Teramo BaSTI (solid lines) and the Padova (dashed lines) isochrones in $V-I$ versus $I$ CMDs at the same solar-scaled composition. Symbols for the RGB tips are same as in Figure 11. It is noted that the Padova RGBs, especially at the older ages $(t \geqslant 5 \mathrm{Gyr})$, are relatively redder than the Teramo BaSTI ones in these CMDs. From the right panels of Figure 12, it is seen that, in general, the Padova TP-AGBs models are more complex than the Teramo BaSTI TP-AGBs. It is further noted from the right panels of Figure 12 that there are discontinuities for the Padova stellar models at the onset of the TP-AGB stage caused by structural changes (Marigo et al. 2008). For instance, the H-exhausted core mass on the TP-AGB starts to increase (and is set to zero before the TP-AGB).

\subsection{Near-IR SBF Models}

Having found that (1) there are significant differences in the $I$-band SBF model predictions using the Padova and the Teramo BaSTI models and (2) the inclusion of TP-AGBs is crucial to match the observations, we now extend our investigation to longer wavelengths, including near-IR SBF models where the effects of the TP-AGB stage are considerably more pronounced.

Figure 13 is similar to the left panel of Figure 10, but here we contrast the $z_{850}$-band SBF models based on the Padova 


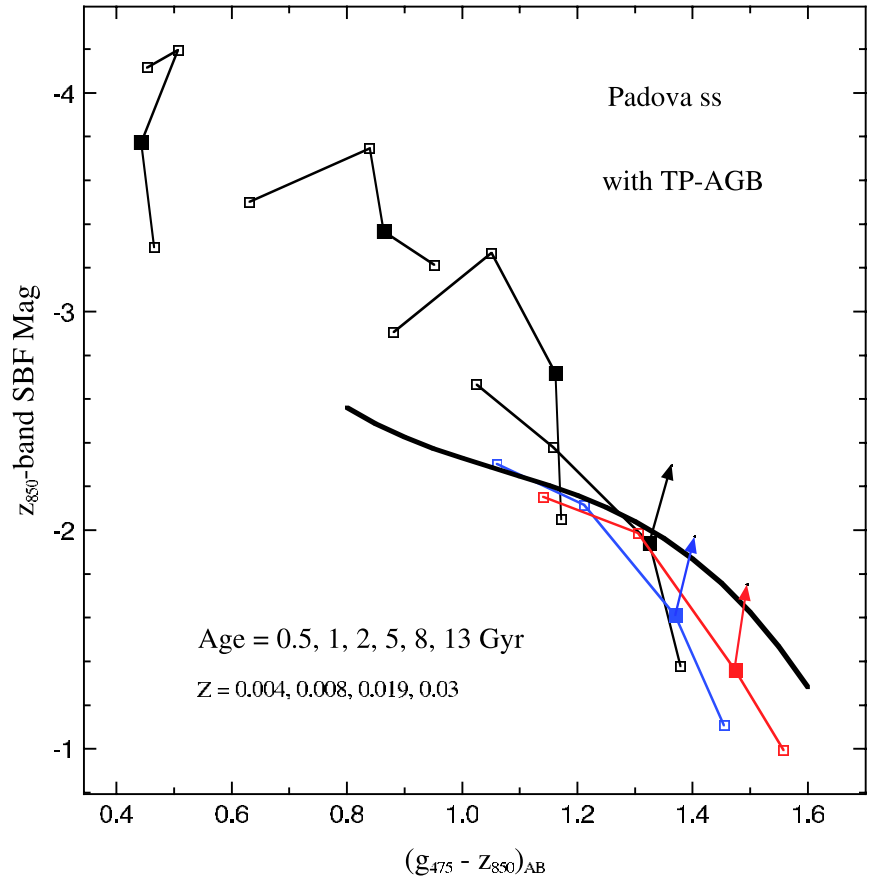

Figure 13. Similar to the left panel of Figure 10, but here we contrast the HST ACS/WFC $z_{850}$-band SBF models employing the Padova stellar models as a function of integrated $g_{475}-z_{850}$ colors at given ages and metallicities. The magnitudes are all in the $\mathrm{AB}$ system. To guide the eye, solar metallicities are marked with filled squares and 8 and $13 \mathrm{Gyr}$ are displayed in different colors. The thick curved line is the empirical relation from Blakeslee et al. (2009). The arrows at 5, 8, and $13 \mathrm{Gyr}$ indicate the estimated $0.4 \mathrm{dex} \alpha$-enhancement effects (see the text).

(A color version of this figure is available in the online journal.)

stellar models ${ }^{15}$ as a function of integrated $g_{475}-z_{850}$ colors at given ages and metallicities. The thick curved line is an empirical relation from Blakeslee et al. (2009). The Hubble Space Telescope (HST) ACS/WFC photometric systems ${ }^{16}$ that we present here are all $\mathrm{AB}$ magnitudes in order to be consistent with the observations. We have converted the Vega magnitude system to $\mathrm{AB}$ magnitude system by employing $\mathrm{F} 475 \mathrm{~W}(\mathrm{AB})=$ $\mathrm{F} 475 \mathrm{~W}$ (Vega) -0.101 and F850LP $(\mathrm{AB})=\mathrm{F} 850 \mathrm{LP}(\mathrm{Vega})$ +0.565 from Sirianni et al. (2005). To guide the eye, solar metallicities are marked with filled squares and 8 and $13 \mathrm{Gyr}$ are displayed in different colors.

The arrows in Figure 13 at 5, 8, and 13 Gyr indicate the estimated effects on the models from 0.4 dex $\alpha$-enhancement. Since the Padova group does not yet provide the matching $\alpha$ enhanced stellar models, those arrows are inferred from the $\alpha$-enhanced $I$-band SBF and integrated $V-I$ model predictions based on the Teramo BaSTI stellar models. We have used the $(V-I)=0.603 \times\left(g_{475}-z_{850}\right)+0.375$ and $I$-band SBF $\mathrm{mag}=0.930 \times z_{850}$-band SBF mag +0.433 . It is interesting to find that the effects of the $\alpha$-enhancement on the $z_{850}$-band SBF models alleviate the mismatches with the observations on the redside, $g_{475}-z_{850}>1.3$. The mismatch that is seen on the blueside in Figure 13, $g_{475}-z_{850}<1.1$ may be relieved if we could employ the BaSTI isochrones as we saw in Figure 10 that the BaSTI isochrones generate systematically much fainter SBF magnitudes compared to Padova isochrones at younger

\footnotetext{
15 The reason that we show the $z_{850}$-band SBF models only based on the Padova stellar models is because the $z 850$-band magnitude is not yet available from the BaSTI Web sites.

${ }^{16}$ The F475W and F850LP are equivalent to $g_{475}$ and $z_{850}$ bands, respectively.
}
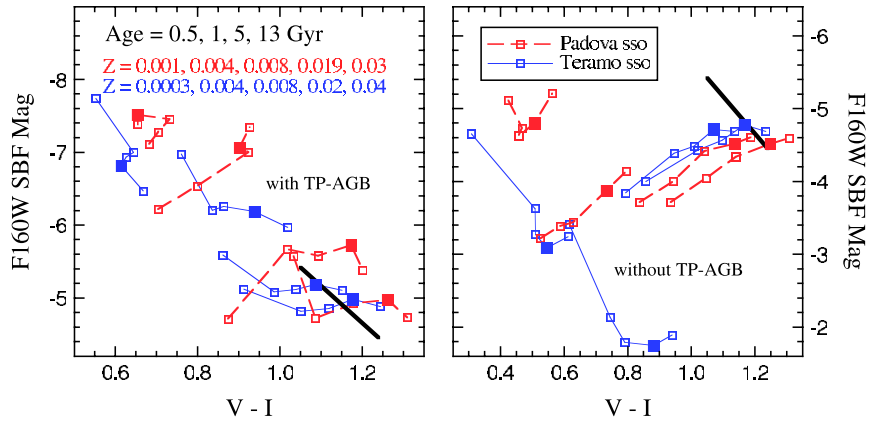

Figure 14. Similar to Figure 10, but here the $H S T$ NICMOS $F 160 W$-band SBF models as a function of integrated $V-I$ colors are contrasted using the Padova (dashed lines) and the Teramo BaSTI (solid lines) stellar models at the same solar-scaled compositions (sso; convective core overshooting is "on"). The thicker line is the empirical sequence from Jensen et al. (2003). The importance of the inclusion of TP-AGB stages is considerably greater here for the near-IR SBF models in order to match the observations. It is noted from the left panel that the predicted near-IR SBF magnitudes become brighter with bluer colors, as observed, when the TP-AGB phase is included. In the right panel, the opposite dependence is seen from the models without the TP-AGB.

(A color version of this figure is available in the online journal.)

ages ( $t<5$ Gyr) with $Z \geqslant 0.004$. Biscardi et al. (2008) provide another interesting comparison of $z_{850}$-band SBF observations with model predictions, but using the Teramo SPoT models. ${ }^{17}$

Figure 14 contrasts the HST NICMOS F160W-band SBF models as a function of integrated $V-I$ colors using the Padova and the Teramo BaSTI stellar models at the same solar-scaled chemical composition. The thicker line is the empirical sequence from Jensen et al. (2003). The HST NICMOS photometric bandpass, $F 160 \mathrm{~W}$, that we present here is Vega magnitudes in order to be consistent with the observations. We have converted $J H K$-band SBF models using the Teramo BaSTI isochrones to $F 160 \mathrm{~W}$-band SBF ones by employing Equation (3) of Jensen et al. (2003). As expected, the importance of the inclusion of the TP-AGB stage is considerably greater here in the nearIR compared to Figure 10. For instance, at $1 \mathrm{Gyr}$ and solar metallicity, the $F 160 \mathrm{~W}$-band SBF magnitude predictions from the Teramo BaSTI models become $\sim 1.8$ mag fainter without TP-AGBs (see Table 3). Also, it is noted that the trend between $F 160 W$-band SBF models and the integrated $V-I$ colors reverses, in the sense that the near-IR SBF magnitudes become brighter with bluer colors when the TP-AGB is included, but fainter at bluer colors when it is omitted. The significant differences between the models based on the Teramo BaSTI and the Padova models are still noticeable even without TPAGBs from the right panel of Figure 14 at the same solar-scaled chemical composition.

Figure 15 is similar to the left panel of Figure 14, but displays the $\alpha$-element enhancement effects for the HST NICMOS $F 160 \mathrm{~W}$-band SBF models based on the Teramo BaSTI. The thick observational fiducial line is the same as in Figure 14. The $F 160 \mathrm{~W}$-band SBF magnitude differences between the solarscaled and the $\alpha$-enhanced models using the Teramo BaSTI stellar models are relatively small, less than 0.2 mag (see Table 3). The reason for the mismatches with the observations at the very red end remains to be resolved. Compared to the observations, the left panel of Figure 4 showed that the $I$-band SBF models are a bit fainter, but here in Figure 15, F160W-band SBF models are brighter. Figure 1 of Blakeslee et al. (2009), however, shows that the very red galaxies become progressively

\footnotetext{
17 http://193.204.1.79:21075/models.html
} 


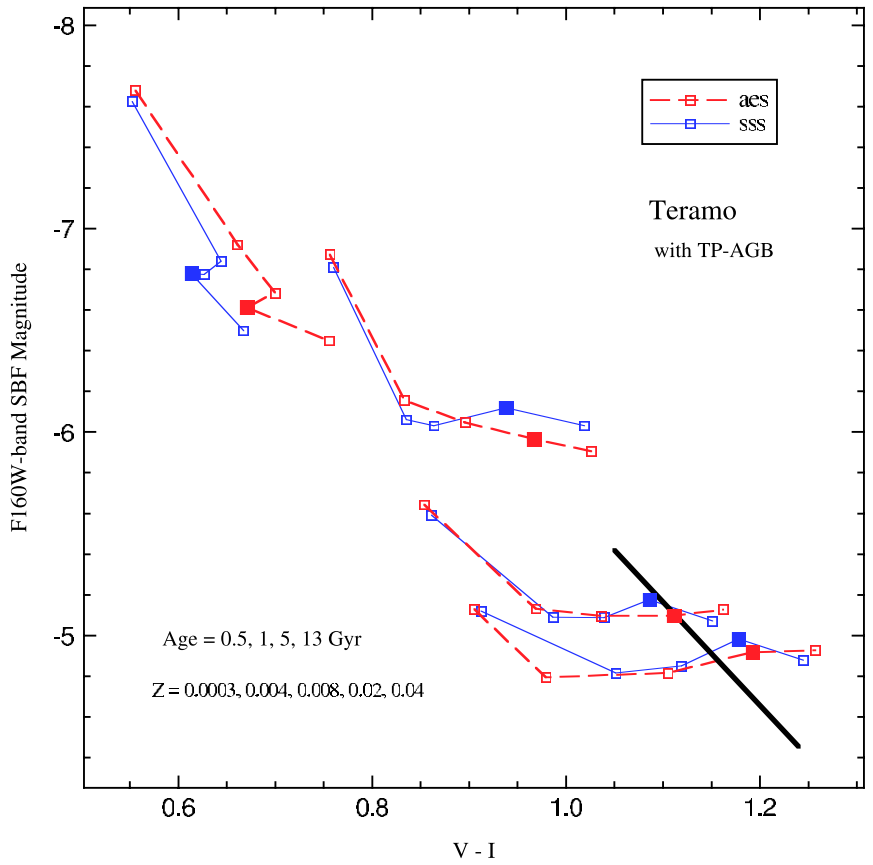

Figure 15. Similar to the left panel of Figure 14, but here the $\alpha$-element enhancement effects are shown for the HST NICMOS F160W-band SBF magnitudes as a function of integrated $V-I$ colors employing the Teramo BaSTI stellar models. Solid lines with squares are solar-scaled models, while dashed lines with squares are $\alpha$-enhanced models. The thick observational fiducial line is same as in Figure 14 . The $F 160 \mathrm{~W}$-band SBF magnitude differences between the solar-scaled and the $\alpha$-enhanced models employing the Teramo BaSTI stellar models are relatively small, less than $0.2 \mathrm{mag}$.

(A color version of this figure is available in the online journal.)

fainter in $z$-band SBF causing the nonlinear relation between SBF magnitudes and the integrated colors as we have seen in Figure 13 .

\section{SUMMARY AND DISCUSSION}

We have presented for the first time the effects of $\alpha$-element enhancement on SBF models and have compared these models with observations. For this purpose, we have employed the Teramo BaSTI Isochrones in this study. In general, we find that the $\alpha$-element-enhanced $I$-band SBF magnitudes are about 0.35 mag brighter and their integrated $V-I$ colors are about 0.02 mag redder mostly because of the oxygen-enhancement effects on the upper RGBs and AGBs. Moreover, the importance of the TP-AGB stages for the I-band and near-IR band SBF magnitudes is illustrated and it becomes clear that TP-AGBs are the indispensable component in order to match the theoretical predictions with the observations. It is also noted that the TPAGBs are more important in the metal-poor regime as shown in Figures 4, 5, 10, and 14 .

We have further shown that the disparity in the SBF model and integrated $V-I$ color predictions is non-negligible when different stellar models are employed as input ingredients even at the same solar-scaled chemical composition. It is our understanding that what causes the disagreement in the integrated $V-I$ colors and $I$-band SBF models using different stellar models are (1) giant branch temperature differences, which occur depending upon the treatment of convection and (2) dissimilar stellar model atmospheres that are employed in order to convert from theoretical planes to observables. Further comparison of SBF model predictions with multiband observations can help illuminate many of the remaining problems in the evolution of bright stars relevant to population synthesis modeling.

The I-band SBF models explored here are slightly fainter than the observations at the red end $(V-I>1.15)$, as shown in Figure 4. A partial solution may come from the nonlinear behavior between galaxy colors in $g_{475}-z_{850}$ and the $z_{850^{-}}$ band SBF magnitudes as illuminated by Blakeslee et al. (2009) in the sense that the red-end galaxies become rapidly fainter in $z_{850}$-band SBF magnitudes. In this context, the $\alpha$-enhanced SBF models help to reconcile the disagreement by making the theoretical $I$-band and $z$-band SBF brighter. Also, rigorous statistical investigations of model isochrones with TP-AGBs that are poorly populated yet very salient because of their prominent luminosity should help alleviate the discrepancies between the observations and the theoretical predictions as suggested by Cerviño et al. (2008), Raimondo (2009), and GonzalezLopezlira et al. (2009). It is evident from the smaller scatter in the observations compared to the theoretical predictions that there is generally less variation among the AGB phases in actual galaxies than may be expected based on the models. The observed relations between SBF magnitudes and integrated colors are very tight, at least for evolved galaxies. The future sophisticated $\alpha$-enhanced SBF studies in various bandpasses should provide some additional constraints on the fine details of the calibration.

We thank Michele Cantiello, Santi Cassisi, Aaron Dotter, Leo Girardi, Paola Marigo, Reynier Peletier, and Gabriella Raimondo for very insightful discussions. We are also grateful to the anonymous referee for her/his careful reading and thoughtful comments that have improved our presentation. Support for this work was provided by the NSF through grant AST-0307487, the New Standard Stellar Population Models (NSSPM) project, and by NASA through grant HST-GO-11083.

\section{REFERENCES}

Bertelli, G., Bressan, A., Chiosi, C., Fagotto, F., \& Nasi, E. 1994, A\&AS, 106, 275 (B94)

Biscardi, I., Raimondo, G., Cantiello, M., \& Brocato, E. 2008, ApJ, 678, 168

Blakeslee, J. P., Lucey, J. R., Tonry, J. L., Hudson, M. J., Narayanan, V. K., \& Barris, B. J. 2002, MNRAS, 330, 443

Blakeslee, J. P., Vazdekis, A., \& Ajhar, E. A. 2001, MNRAS, 320, 193 (B01)

Blakeslee, J. P., et al. 2009, ApJ, 694, 556

Bruzual, G., \& Charlot, S. 2003, MNRAS, 344, 1000 (BC03)

Cantiello, M., Raimondo, G., Brocato, E., \& Capaccioli, M. 2003, AJ, 125, 2783

Cassisi, S., Salaris, M., Castelli, F., \& Pietrinferni, A. 2004, ApJ, 616, 498

Cerviño, M., Luridiana, V., \& Jamet, L. 2008, A\&A, 491, 693

Chabrier, G. 2003, PASP, 115, 763

Coelho, P., Bruzual, G., Charlot, S., Weiss, A., Barbuy, B., \& Ferguson, J. W. 2007, MNRAS, 382, 498

Cordier, D., Pietrinferni, A., Cassisi, S., \& Salaris, M. 2007, AJ, 133, 468

Dotter, A., Chaboyer, B., Ferguson, J. W., Lee, H.-C., Worthey, G., Jevremović, D., \& Baron, E. 2007a, ApJ, 666, 403

Dotter, A., Chaboyer, B., Jevremović, D., Baron, E., Ferguson, J. W., Sarajedini, A., \& Anderson, J. 2007b, AJ, 134, 376

Dotter, A., Chaboyer, B., Jevremović, D., Kostov, V., Baron, E., \& Ferguson, J. W. 2008, ApJS, 178, 89

Ferguson, J. W., Alexander, D. R., Allard, F., Barman, T., Bodnarik, J. G., Hauschildt, P. H., Heffner-Wong, A., \& Tamanai, A. 2005, ApJ, 623, 585

Ferraro, F. R., Origlia, L., Testa, V., \& Maraston, C. 2004, ApJ, 608, 772

Freedman, W. L., et al. 2001, ApJ, 553, 47

Gonzalez-Lopezlira, R. A., Bruzual, G., Charlot, S., Ballesteros-Paredes, J., \& Loinard, L. 2009, MNRAS, in press (arXiv:0908.4133)

Iben, I. Jr, \& Renzini, A. 1983, ARA\&A, 21, 271

Jensen, J. B., Tonry, J. L., Barris, B. J., Thompson, R. I., Liu, M. C., Rieke, M. J., Ajhar, E. A., \& Blakeslee, J. P. 2003, ApJ, 583, 712

Kim, Y.-C., Demarque, P., Yi, S. K., \& Alexander, D. R. 2002, ApJS, 143, 499 Kroupa, P. 2001, MNRAS, 322, 231 
Lee, H.-C. 2001, PASP, 113, 1021

Lee, H.-C., \& Worthey, G. 2005, ApJS, 160, 176

Lee, H.-C., Worthey, G., \& Blakeslee, J. P. 2007a, in IAU Symp. 241, Stellar Populations as Building Blocks of Galaxies, ed. R. F. Peletier \& A. Vazdekis (Cambridge: Cambridge Univ. Press), 187

Lee, H.-C., Worthey, G., Trager, S. C., \& Faber, S. M. 2007b, ApJ, 664, 215

Lee, H.-C., et al. 2009, ApJ, 694, 902

Liu, M. C., Charlot, S., \& Graham, J. R. 2000, ApJ, 543, 644

Maraston, C. 2005, MNRAS, 362, 799 (M05)

Maraston, C., Daddi, E., Renzini, A., Cimatti, A., Dickinson, M., Papovich, C., Pasquali, A., \& Pirzkal, N. 2006, ApJ, 652, 85

Marigo, P., Girardi, L., Bressan, A., Groenewegen, M. A. T., Silva, L., \& Granato, G. L. 2008, A\&A, 482, 883

Marín-Franch, A., \& Aparicio, A. 2006, A\&A, 450, 979 (MA06)

Mei, S., Quinn, P. J., \& Silva, D. R. 2001, A\&A, 371, 779

Mei, S., et al. 2005, ApJ, 625, 121

Mieske, S., Hilker, M., \& Infante, L. 2006, A\&A, 458, 1013

Mouhcine, M., González, R. A., \& Liu, M. C. 2005, MNRAS, 362, 1208

Mucciarelli, A., Origlia, L., Maraston, C., \& Ferraro, F. R. 2009, ApJ, 690, 288

Percival, S. M., Salaris, M., Cassisi, S., \& Pietrinferni, A. 2009, ApJ, 690, 427 (P09)

Pietrinferni, A., Cassisi, S., Salaris, M., \& Castelli, F. 2004, ApJ, 612, 168 (P04)

Pietrinferni, A., Cassisi, S., Salaris, M., \& Castelli, F. 2006, ApJ, 642, 797
Raimondo, G. 2009, ApJ, 700, 1247

Raimondo, G., Brocato, E., Cantiello, M., \& Capaccioli, M. 2005, AJ, 130, 2625 $(\mathrm{R} 05=\mathrm{SPoT})$

Reimers, D. 1975, Mem. R. Soc. Liege Ser., 8, 369

Salaris, M., Chieffi, A., \& Straniero, O. 1993, ApJ, 414, 580

Salasnich, B., Girardi, L., Weiss, A., \& Chiosi, C. 2000, A\&A, 361, 1023

Salpeter, E. E. 1955, ApJ, 121, 161

Scalo, J. M. 1998, in ASP Conf. Ser. 142, The Stellar Initial Mass Function, ed. G. Gilmore \& D. Howell (San Francisco, CA: ASP), 201

Schiavon, R. P. 2007, ApJS, 171, 146

Sirianni, M., et al. 2005, PASP, 117, 1049

Thomas, D., Maraston, C., \& Bender, R. 2003, MNRAS, 339, 897

Tonry, J. L. 1991, ApJ, 373, L1

Tonry, J. L., Blakeslee, J. P., Ajhar, E. A., \& Dressler, A. 1997, ApJ, 475, 399

Tonry, J. L., Blakeslee, J. P., Ajhar, E. A., \& Dressler, A. 2000, ApJ, 530, 625

Tonry, J. L., Dressler, A., Blakeslee, J. P., Ajhar, E. A., Fletcher, A. B., Luppino, G. A., Metzger, M. R., \& Moore, C. B. 2001, ApJ, 546, 681

Vandenberg, D. A., Bergbusch, P. A., \& Dowler, P. D. 2006, ApJS, 162, 375

Worthey, G. 1993, ApJ, 409, 530

Worthey, G. 1994, ApJS, 95, 107 (W94)

Worthey, G. 1998, PASP, 110, 888

Worthey, G., Faber, S. M., \& González, J. J. 1992, ApJ, 398, 69

Yi, S. K. 2003, ApJ, 582, 202 